\title{
An Application of Artificial Neural Network for Wind Speeds and Directions Forecasts in Airports
}

\author{
E. Lawrence, E. J. Garba, Y. M. Malgwi, and M. A. Hambali
}

\begin{abstract}
Wind speed patterns are highly dynamic and non-linear and thus cannot be accurately forecasted using conventional linear regression models. In this work, Artificial Neural Network (ANN) technique was applied to forecast wind speeds and directions in airports. Monthly data of maximum temperature, minimum temperature, wind speed, wind direction, relative humidity and wind run for Yola International Airport were collected from 1995 to 2021 from Nigerian Meteorological Agency (NIMET) Abuja-Nigeria. Six Neural Network models were built. ANN with no hidden layers, ANN model with one hidden layer and two dropout layers, ANN model with four hidden layers and three dropout layers, ANN model with eight hidden layers, ANN model with nine hidden layers and finally, ANN model with ten hidden layers. Back Propagation training algorithm was implemented using the PYTHON toolbox. Each of the models was trained using the training dataset and validated using the validation dataset. To test the forecasting ability of each of the models we tested it using unknown data that is the test dataset. The results from each of the models were organized and assessed in terms of the magnitude of the statistical error between the measured result and the real data. This was achieved by measuring the average of the Mean Square Errors (MSE) and Mean Absolute Error (MAE) for each of the models used for forecasting both wind speeds and directions. The results show that Multilayer perceptron with ten hidden layers with $(\mathrm{MSE})=0.92$ and $($ MAE $)=0.73$ emerged as the most preferred model for wind speeds forecast while the multilayer perceptron with four hidden layers with $($ MSE) $=1,858$ and $($ MAE $)=35$ emerged the most preferred model for wind directions forecast. Future research can be carried out to improve the accuracy of the model for wind direction forecasts.
\end{abstract}

Keywords - Artificial Neural Network, Airport operation, Forecasting, Back-propagation, Wind speeds and directions.

\section{INTRODUCTION}

The wind can be referred to as moving air which is difficult to control because of its irregular or dynamic nature. Its source comes from the pressure gradient between different regions caused by the unsteady heating of the sun on the earth surface [1]. In addition, a complicated event such as rotation of the earth, physical effects of the

\footnotetext{
Submitted on January 15, 2022.

Published on February 14, 2022.

E. Lawrence, Computer Science Department, Federal University Wukari, Taraba, Nigeria.

(corresponding e-mail: lawrence@fuwukari.edu.ng)

E. J. Garba, Computer Science Department, Modibbo Adama University, Yola, Nigeria.

(e-mail: e.j.garba@mautech.edu.ng)

Y. M. Malgwi, Computer Science Department, Modibbo Adama University, Yola, Nigeria

(e-mail: yumalgwi@mautech.edu.ng)

M. A. Hambali, Computer Science Departments, Federal University

Wukari, Taraba-Nigeria.

(e-mail: hambali@fuwukari.edu.ng)
}

mountain, obstruction and roughness of the terrain can determine the behaviour of the wind. Generally, the influence of obstacles and roughness decreases as a function of the height above the ground that is, the higher a region above the ground, the greater the wind speed at any given moment [2].

Wind speeds and directions are the two most important weather parameters that affect airport operations. These two parameters need to be forecasted because coincidently, they can represent local topography effects non- resolved by weather models [3]. Furthermore, it can be very difficult to predict wind speeds using simple methods or techniques but if eventually it can be predicted with high accuracy especially in wind power plants, more useful information can be provided to the power companies to help in scheduling power generation. Thus, allowing efficient use of Wind energy [3].

So also, in airports, if wind speeds and directions can be forecasted with high accuracy, it will greatly help in the safety of the aircraft during take-off and landing.

According to Rotich [4], Wind speeds and directions can be useful in electricity generation, Marine engineering, electricity load forecasting and aviation. He went further to say that, wind turbine pull up energy from a moving mass of air that is wind and converts its kinetic energy into mechanical energy from which the generator or alternator changes it into the electrical energy we know today; and for aviation he said, wind speeds and directions can affect aircraft safety during landings and take-offs.

Airports are places or areas, with particular wind regimes favourable to air traffic where aircraft takes off and land. The wind behaviour can be affected by the surrounding topography by blocking, increasing, or changing its direction as it travels which generate local wind effects not resolved by numerical weather prediction (NWP) [5]. To ensure a safe operation of aircraft in airports, airport terminal wind information is very important especially during take-off or landing and for managing airport runway configurations. Because every aircraft take-off and land in the wind, two components affect aircraft during take-off and landing; These components are headwind/tailwind component which affects aircraft vertical control, takeoff/landing distance, and in-trail separation, and the crosswind component which affects lateral control of the aircraft, especially during take-off, approach, and landing [6]. Crosswinds can greatly affect aircraft control during taxing and ramp operations. Every aircraft have certain limits for wind performance provided by the manufacturers, and also there are policies established for the pilots that define the limits for take-off and landing. Because aircraft vary by size and design, so also their wind requirements may be adjusted for specific runway conditions. 
According to Clark et al. [6], after the second world war, commercial aviation and its fellow airports experienced the fastest growth and construction which was followed by a proliferation of airport wind/weather sensor deployment that would continue throughout the latter half of the 20th Century this is because, limitation of wind data and other factors such as lack of proper forecast of the wind speeds and directions and communicating of the results which is the airport wind information to the flight crew will cause problems to the aircraft, especially during landing and takeoff. Generally, an aircraft is affected by the change in wind direction/velocity because the wind also changes the aircraft motion relative to the ground.

Wind speeds and directions, the forecast can be divided into the four-time horizon, this includes; very short, short, medium and long term. Very short-term time horizon is for very few minutes only and these forecasts are useful for landing and take-off of the aircraft, turbine control and unit commitment in the power sector. Short term time horizon could be for 30 minutes to 6 hours; these forecasts are useful for preload sharing. The medium-term time horizon is for 6 hours to 24 hours. These forecasts are useful for power system management and energy trading. Long term time horizon is for one day to seven days. These forecasts are useful for the maintenance planning of conventional power generation plants [7].

Forecasting becomes an essential factor when the penetration of wind speed increases. Accurate forecasting reduces the risk of uncertainties in airports and power systems. Different research works have been carried out to develop predictive models; this can be found in the literature. The approaches found in the literature may include physical methods, statistical methods, hybrid of physical and statistical models, artificial intelligence and other new methods. Statistical methods, such as autoregressive integrated moving average models, make forecasts by finding the relationship of the observed wind speed time series. In the literature, artificial intelligence techniques such as neural networks, fuzzy logic, etc. are found to be more accurate compared to traditional statistical models [8]. Often, both physical and statistical models are used together, where results obtained from NWF serves as input variables, together with historical data, to train the system on the local conditions according to statistical theories. Lately, there are new methods based on artificial intelligence techniques that have been developed, these include; the ANN of multi-layer perceptrons, radial basis function, recurrent neural networks and fuzzy logic. Artificial Intelligence approaches normally use artificial neural networks (ANN) where the processing of information in the network is carried out through calculations that have been internally determined from a training period of available data [8].

Artificial neural networks are network system which is built by imitating the learning behaviour of human being. The fundamental processing elements are the neurons, which are placed in serial layers with three components: the input layer, the hidden layer and the output layer [9]. The data get into the network from the input layer to the output layer through the hidden layer. Neural networks are helpful when it is necessary to simulate the behaviour of systems characterized by noisy and incomplete data. Neural networks learn by example and they cannot be programmed to perform a specific task [10].

In this present work, artificial neural network methodology was applied for the forecast of wind speeds and directions in airports for the safety of aircraft during landing and take-off. Meteorological data were collected from the Nigeria Meteorological Agency (NIMET) Abuja for Yola international airport Adamawa state. Six neural network models were built. Back propagation training algorithm was implemented using the python toolbox. Just as explained above, there are various methods proposed by different researchers, but why we chose ANN over other methods of forecasting is because first, there is no requirement for any assumptions, second is draw from historic data to generate forecasts and lastly, it is efficient enough in solving the complex non-linear problems successively. According to Esen, Inalli, Sengur and esen,[11], the advantage of ANN over other models is their ability to model a multivariable problem given by the complex relationships between the variables and can extract the implicit non-linear relationships among these variables employing 'learning' with training data.

\section{OVERVIEW OF ARTIFICIAL NEURAL NETWORK (BIOLOGICAL NEURON)}

The human brain consists of billions of cells that are connected to pass or process information. These interconnected cells are called (neurons) which make up the neural network of the brain [12]. A neuron is the basic processing unit in the neural network, it has four components namely: the dendrites, the soma (cell body), the axon and the synapse. A soma contains a cell nucleus, Dendrites branch into a bushy network around the cell to receive input from other neurons, and the axon stretches out for a long-distance, typically a centimetre and as far as a meter in extreme cases. The axon is an output channel to other neurons; it branches into strands and sub-strands to connect to the dendrites and cell bodies of other neurons [13]. The synapse is the connecting junction of the network. Just as the biological neuron has four components, the artificial neuron also has three main components that comprise: set of input connections that bring inactivation from other neurons, a processing unit that sums the inputs and then applies a non-linear activation and finally, an output line that transfers the result to other neurons. Therefore, an artificial neural network is a mathematical model of the brain that learns (weights adjustment) by using the learning algorithm known as back-propagation.

\section{RELATED WORKS}

Wesonga, Nabugoomu, Ababneh, and Owino [14], in their work, assessed the autoregressive integrated moving average (ARIMA) and artificial neural network (ANN) models on the wind speed time series problem. Data on wind speed and minimum and maximum temperatures were evaluated. Wind speed was established to follow a time series that fluctuated around $\operatorname{ARIMA}(0,1,1)$ and ARIMA 
$(1,1,1)$. The optimal ANN model was established at 10 hidden neurons. From the performance of the models, it shows that the ANN wind speed model was better than the ARIMA model. The result also shows that wind speed prediction accuracy can be improved to secure the safety of air traffic flow as well as support the implementation of a reliable and secure power generation system at the airport.

Larraondo et al. [2], presented a model for improving the accuracy of airport weather forecasts produced by numerical weather prediction (NWP), this model learns from the relationships between previously modelled and observed data. Their model was based on a new machine learning methodology that allows circular variables to be naturally incorporated into regression trees. The result shows that the model produced more accurate results than linear and previous circular regression tree methodologies.

Kaur, Kumar and Segal [15] implemented five different neural network models based on historical time series. The result shows that the neural network with 19 hidden layers, 4 inputs and 1 output performs much better than the other models for short term wind speed forecasting.

Fazelpour, Tarashkar, and Rosen [16] proposed four hybrid models such as ANN-PSO, ANFIS, ANN-GA, NNRBF. Out of these models, the results showed that the hybrid model PSO gives better accuracy.

$\mathrm{Hu}$, Zhang, Yu, and Xie [17], proposed a new hybrid model namely HGN-support vector regression. The model was compared with six methods and the results show that the proposed method provides better accuracy.

Rozas-Larraondo, et al., [5], studied a new method based on nonparametric multivariate locally weighted regression for improving the forecasted wind speed of a numerical weather model. In their study, wind direction data were used to build different regression models as a way of accounting for the effect of the surrounding topography. Their studies show that the use of this technique offers similar levels of accuracy for wind speed forecasts compared with other machine learning algorithms with the advantage of being more intuitive and easier to interpret.

Hussin, Yusuf, and Norrulashikin, [18], used the Autoregressive Integrated Moving Average (ARIMA) and ARIMA-GARCH models to forecast future wind speed series. The Ljung-Box test was used to determine the presence of serial autocorrelation, while the Engle's Lagrange Multiplier (LM) test was used to investigate the presence of Autoregressive Conditional Heteroscedasticity (ARCH) effect in the residual of the ARIMA model. In their work, three stations showed good fit using the ARIMA modelling since no successive correlation and ARCH effect were present in the residuals of the ARIMA model, while the ARIMA-GARCH had proven to accurately capture the non-linear characteristic of the wind speed daily series for the remaining stations. They used root mean square error (RMSE) and mean absolute percentage error (MAPE) to measure the performance of the models, and the results showed that both ARIMA and ARIMA-GARCH models proposed provided good forecasts accuracy measure of wind speed series in Peninsular Malaysia.

Liu et al. [19], used two hybrid forecasting frameworks the Wavelet-Genetic Algorithm (GA)-Multilayer Perceptron (MLP) and the Wavelet-Particle Swarm Optimization
(PSO)-Multilayer Perceptron (MLP) to predict nonstationary wind speeds. In this paper, the activation function used for the hidden layers and output layers were the 'tan h' function and 'linear' function respectively. The authors affirmed that both the Wavelet-GA-MLP and the WaveletPSO-MLP hybrid forecasting frameworks provided suitable forecasts.

Shi, Guo, and Zheng [20], proposed a hybrid approach for forecasting both wind speed and power generation time series in Colorado and North Dakota of USA. In this study, the authors used two hybrid models such as ARIMA-ANN hybrid model and ARIMA-SVM hybrid model. They used data from 2005 to December 2007 in Colorado and North Dakota to train and test the models. They used the mean absolute error (MAE) and the root means square error (RMSE) to measure the performance of the proposed model. The results showed that the developed hybrid models performed better than the single ARIMA and SVM methods.

Jung and Kwon [21], applied two ANN based on weighted error functions to improve the accuracy of the long-term energy estimation. In this study, the researchers used actual wind speed and direction parameters measured from the four sites. The data collected for the study ranges from 1st October 2007 to 30th September 2009. The algorithm used for the study was Levenberg-Marquardt algorithm and the activation functions used for the training of the networks were the sigmoid and tanh activation functions. The performance of the proposed ANNs was compared with conventional ANNs. The results showed that the proposed ANNs performed better than the conventional ANNs.

\section{Methodology}

\section{A. Data}

Monthly data of maximum temperature, minimum temperature, wind speed, wind direction, relative humidity and wind run for Yola International Airport were collected from 1995 to 2021 from Nigerian Meteorological Agency (NIMET) Abuja-Nigeria. We have a total data point of 318 but wind-run is missing from 1995-2015 so, we took the median of the values we have for wind-run which is from 2016-2021 and used it to populate the missing values that are, 1995-2015. The data was divided into $60 \%$ and $40 \%$ for the train and test set. The test set was further divided into $50 \%$ test and $50 \%$ validation. We used the MinMaxScaler algorithm to normalize the data.

In this study, we applied Artificial Neural Network (ANN) technique to forecast wind speeds and directions in airports. ANN and machine learning (ML) are both aspects of cognitive science that evolved from two concepts of pattern recognition and computational learning, which are components of Artificial Intelligence (AI). A backpropagation algorithm was also implemented for the training of the network; rectified linear unit(RELU) was used for the activation function and Python programming language and leveraging the Tensor flow library were used for training and forecasting the wind speeds and directions. 


\section{B. Construction of the Predictive Model Using ANN}

Six neural network models were built: ANN model with no hidden layer, ANN model with one hidden layer and two dropout layers, ANN model with four hidden layers and three dropout layers, ANN model with eight hidden layers, ANN model with nine hidden layers and finally, ANN model with ten hidden layers were built using the python programming language and leveraging the Tensor flow library. The input layer was formed as a matrix of a meteorological time series data multiplied by weight coefficient $w k$ that is obtained by the learning algorithm in the training process.

\section{Model Validation with Train Dataset}

The model was validated with an earlier prepared set of the input and output data for that purpose (20\% of data) to compare the model response with the measured data. The model response based on the validation data set has to be evaluated graphically and by applying numerical quality measures which are going to be appraised according to each used numerical model quality criteria. Therefore, we used at least two numerical quality measures to measure performance: (i) Mean Absolute Error (MAE) and (ii) Mean Square Error (MSE).

\section{Model Evaluation with the Test Dataset}

The model was evaluated with an earlier prepared set of the input and output data for that purpose (20\% of data) to compare the model response with the measured data.

\section{RESULTS}

This section presents the results of the experiments carried out. Table I shows the results of wind speeds and directions forecast for the six models built. Figures 1-6 compare the results of six models built by showing their regression line.

TABLE I: SUMMARY OF THE RESUlTS OF WIND SPEEDS AND DIRECTIONS FORECAST USING SINGLE PERCEPTRON WITH NO HIDDEN LAYER, ONE HIDDEN LAYER, FOUR HIDDEN LAYERS, EIGHT HIDDEN LAYERS, NINE HIDDEN LAYERS AND TEN HIDDEN LAYERS RESPECTIVELY

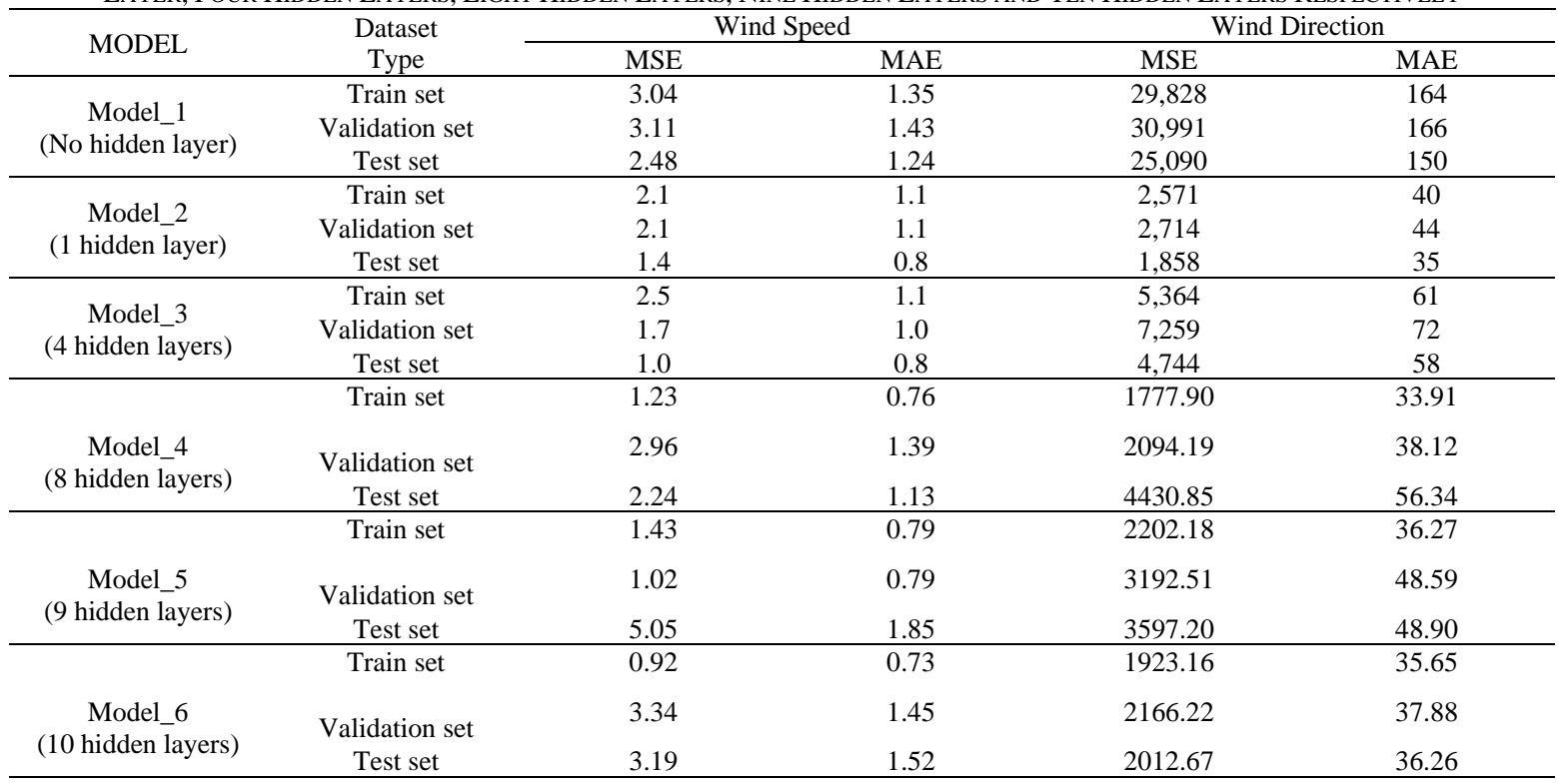
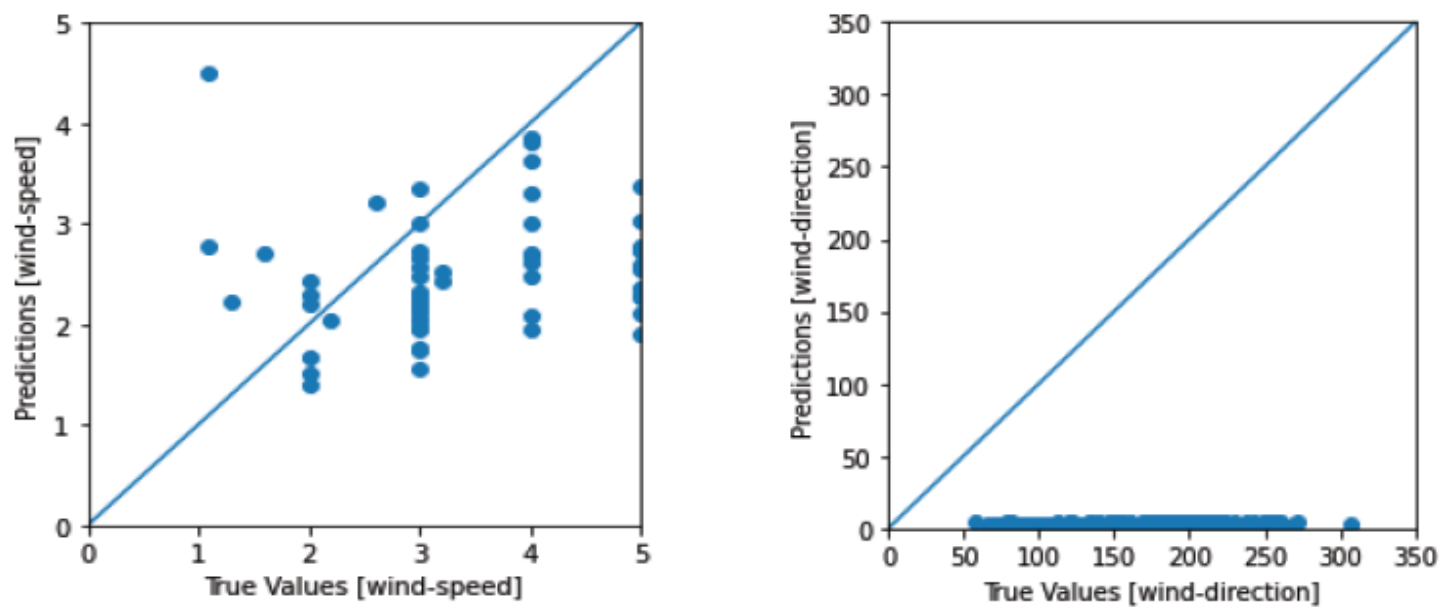

Fig. 1. Prediction of wind speed and direction for Model_1. 

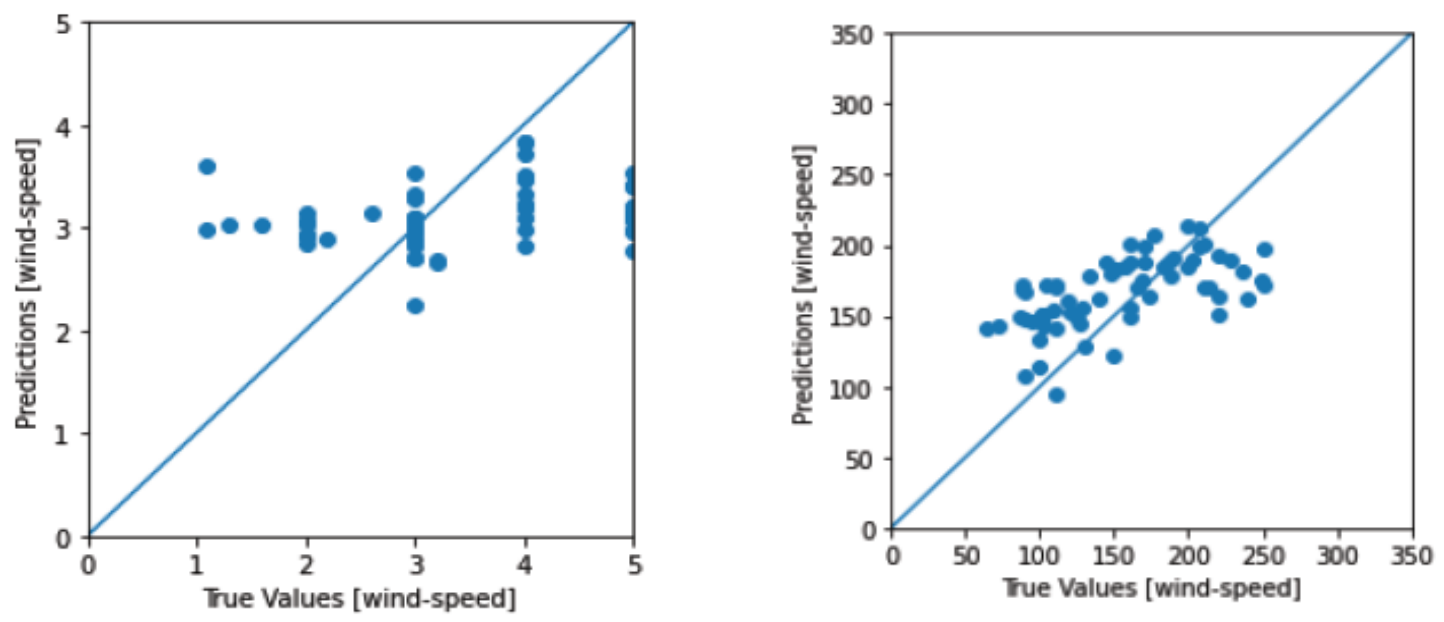

Fig. 2. Prediction of wind speed and direction for Model_2.
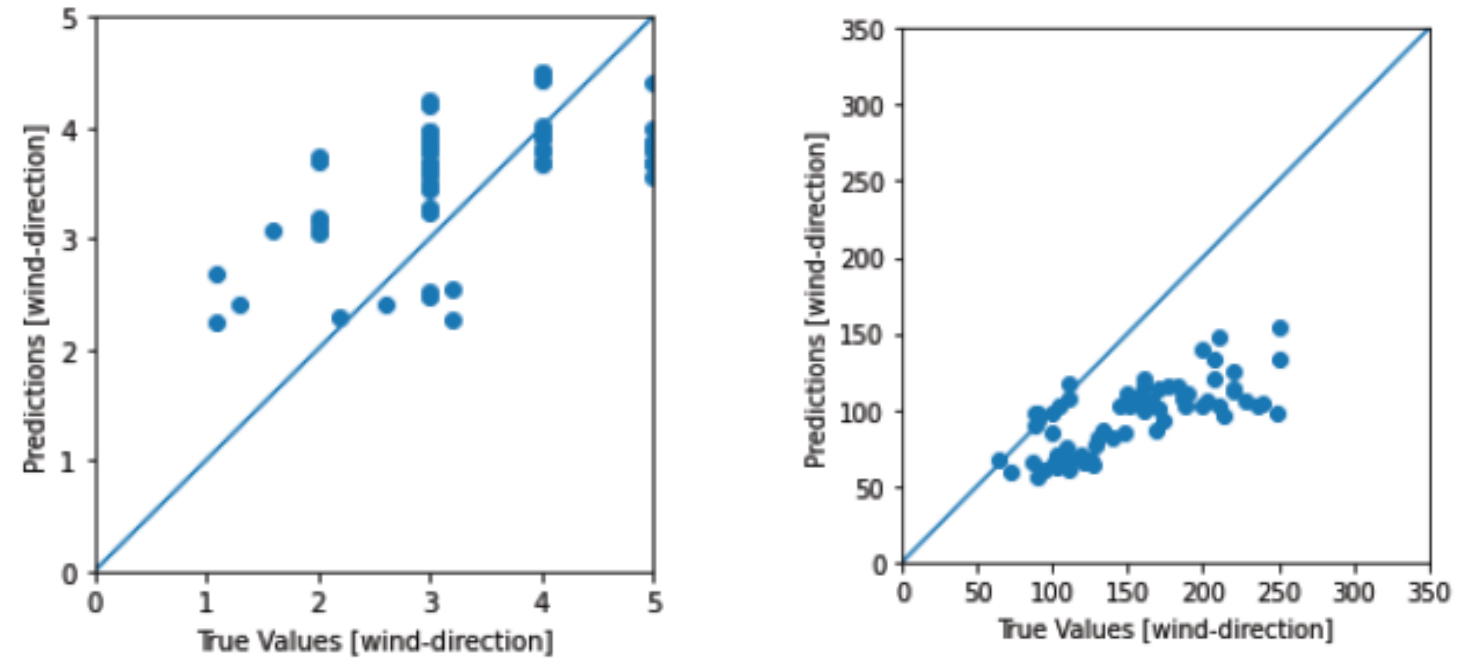

Fig. 3. Prediction of wind speed and direction for Model_3.
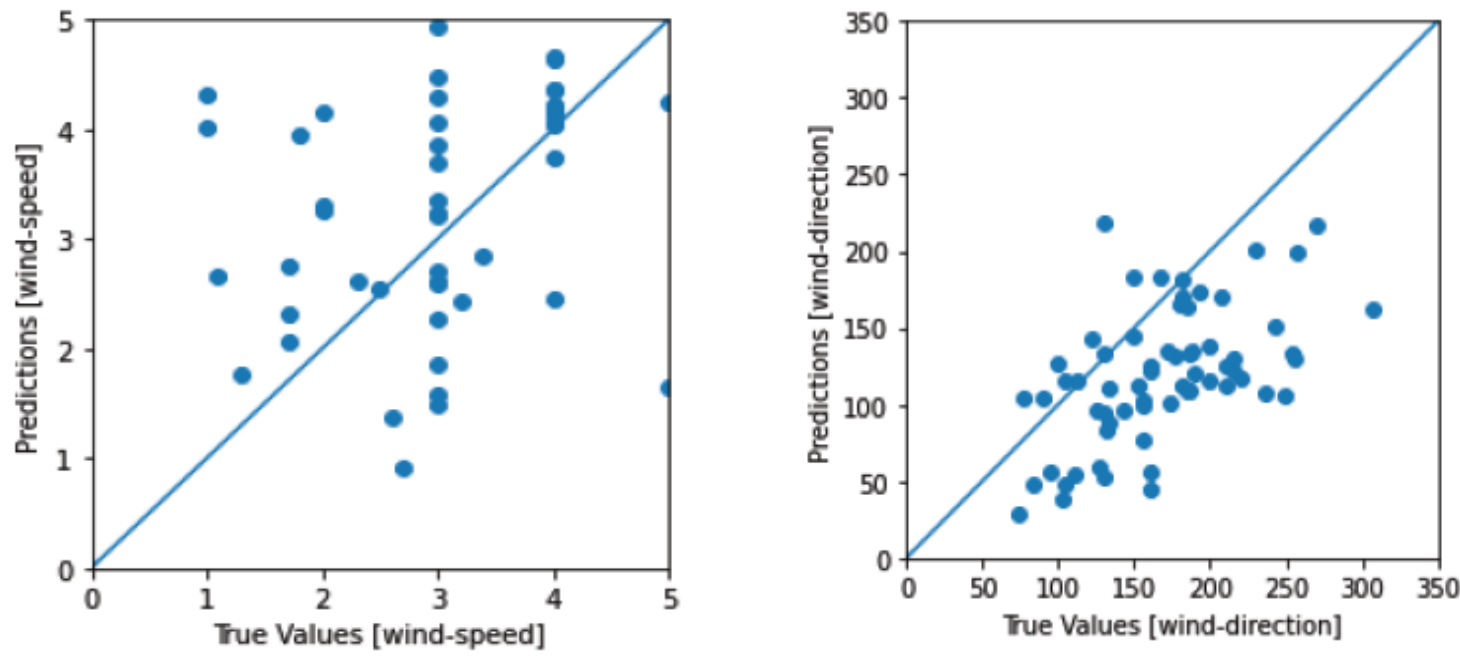

Fig. 4. Prediction of wind speed and direction for Model_4. 

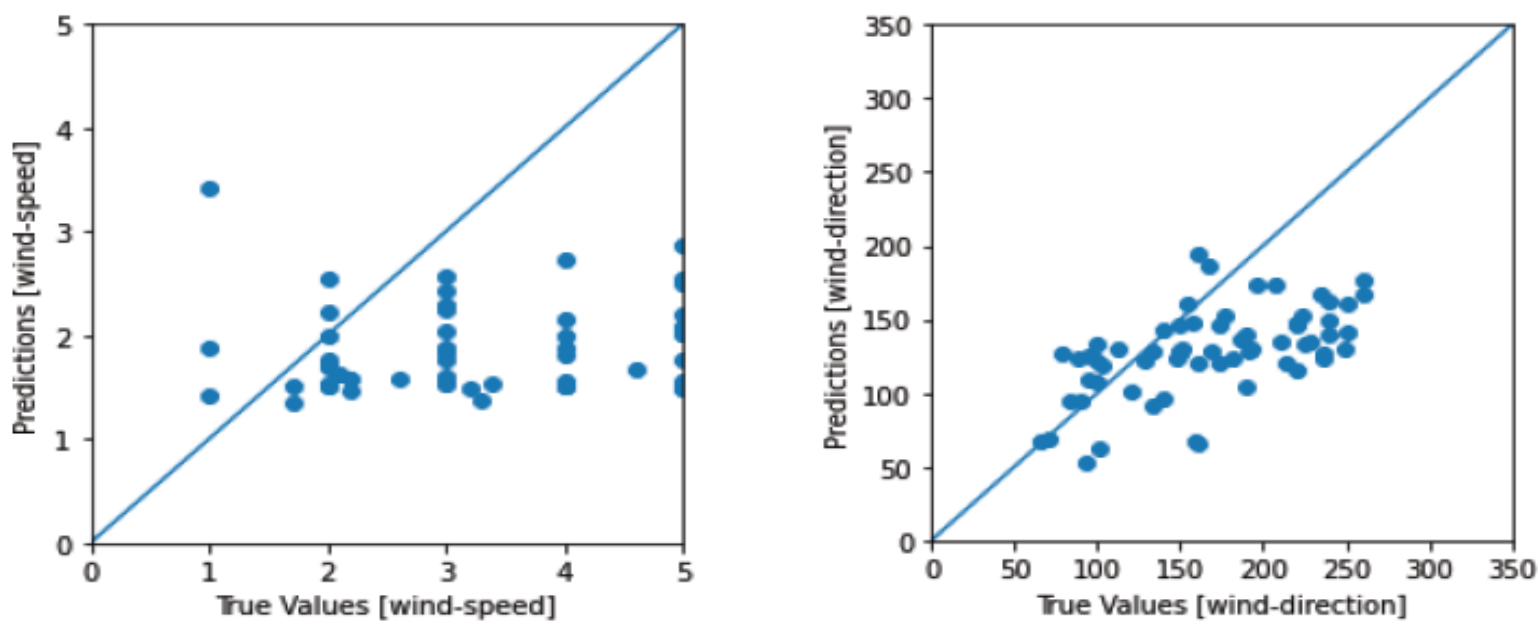

Fig. 5. Prediction of wind speed and direction for Model_5.
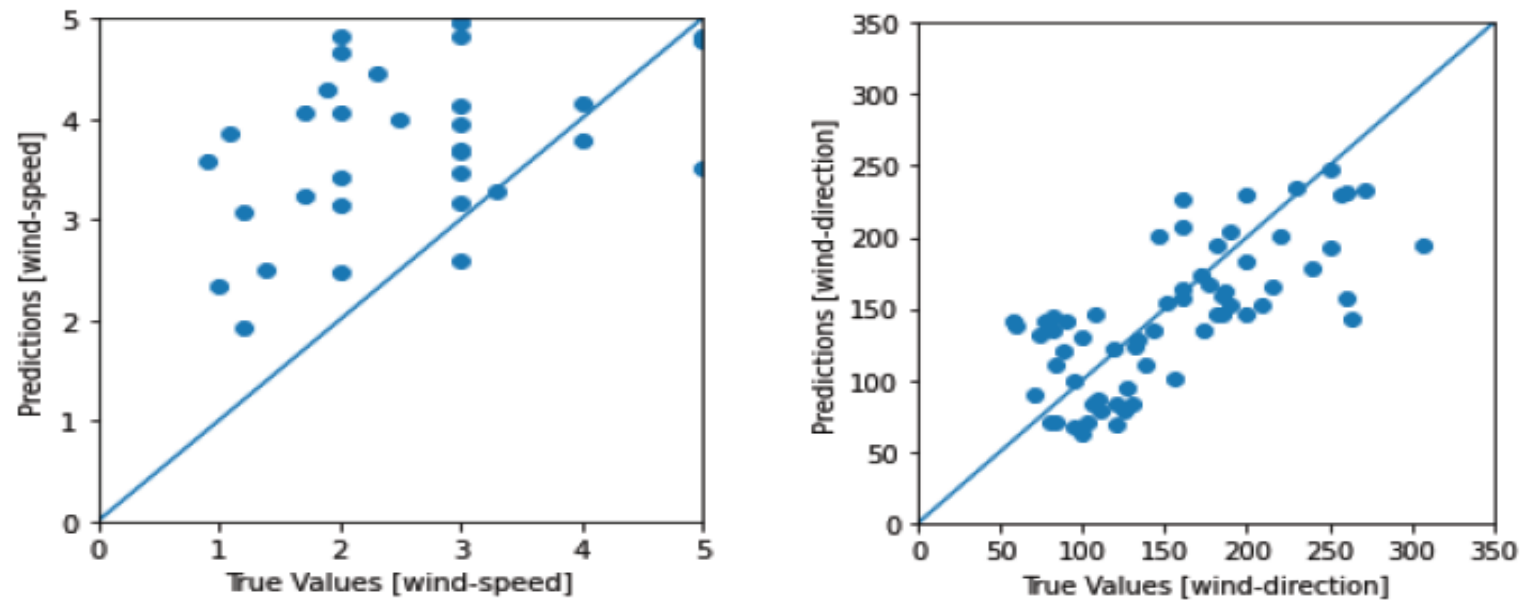

Fig. 6. Prediction of wind speed and direction for Model_6.

\section{DISCUSSION}

The dataset was divided into $60 \%, 20 \%$ and $20 \%$ for training, validation and testing respectively. Six artificial neural networks (ANN) models were built for the study. Each of the models was trained using the training dataset and validated using the validation dataset. To test the forecasting ability of each of our models we tested it using unknown data that is the test dataset. The results from each of the models were organized and assessed in terms of the magnitude of the statistical error between the measured result and the real data. This was achieved by measuring the average of the Mean Square Errors (MSE) and Mean Absolute Error (MAE) for each of the models used for forecasting both wind speeds and directions. Machine learning involves lots of experimentation and hyperparameter tuning for that purpose, we ran the entire codebase at least 50 times using different activation functions, hidden layers, optimization functions, number of neurons and epoch just to see what combination performs better. From Table I, we can see that model_1 performed poorly due to the high error values, this is because it has no hidden layers so it could only measure linearly separable datasets. The result shows that model_1 performed poorly in both the training set and test set. In model_2, the errors in the wind speed decrease but in model_3 increase in train set while the validation and test set reduce. In the wind direction, the errors decrease from model 1 to model 2 and increase in model_3. In model_4 both the wind speed and wind direction errors decrease in the train set but increase in the test set. In model_5, the wind speed errors increase both in the train and test set while in the wind direction, the error increase in the train set but a decrease in the test set. In model_6, the wind speed errors decrease both in the train set and the test set while in the wind direction, both the train and test set decrease from model_5 to model_6. From the analysis, we may conclude that the wind speeds have their optimal value at model_6 in the train set and model_3 in the test set. While the wind directions have their optimal value at model_2 both in train and test set. Therefore, model_6 with $(\mathrm{MSE})=0.92$ and $(\mathrm{MAE})=0.73$ emerged the most preferred model for wind speeds forecast while model_2 with $(\mathrm{MSE})=1,858$ and $(\mathrm{MAE})=35$ emerged the most preferred model for the wind direction forecast. From the results, there is no doubt that the value for the MSE for wind direction is still very high. This could be the result of bad and insufficient data. Therefore, this gives room for future research to improve the accuracy of the wind direction model.

\section{CONCLUSION}

Our study has shown that wind speeds and directions are an obstruction to the smooth flow of air traffic in airports; this is because of every aircraft take-off and land in the air. So to have a smooth flow of air traffic and for the safety of 
the aircraft, these need to be forecasted. Various types of artificial neural networks used for different purposes have been developed over time and at the moment they have been implemented in several computer programming languages which make them easy to construct and use. In this study, six types of neural network models were built using the python programming language and leveraging the Tensor flow library. These tools were used for training and forecasting wind speeds and directions.

Quantitatively, based on the models' forecasting accuracy, and by using the MSE and MAE statistics, the ANN model with ten hidden layers emerged as the most preferred model for forecasting wind speeds while ANN model with four hidden layers emerged as the most preferred model for wind directions forecast. The ANN model with one hidden layer also produced highly accurate predictions and fewer generalization errors compared to the ANN model with no hidden layer. The ANN model with no hidden layer was unable to model our data and failed to deliver an accurate forecast due to its highly linear architecture.

To obtain good results with neural networks, data quantity is as important as data quality. A large amount of data is needed for the training of the model. Our models were trained and tested using a monthly dataset collected from 1995 - 2021 with one parameter missing. There is no doubt, that a good and sufficient dataset will produce a more accurate forecast and fewer generalization errors, and a bad and insufficient dataset will produce inaccurate forecast and large errors. Also, better models could be built by adding more hidden layers even though, not in all cases. Models produced by adding more hidden layers would have to implement regularization techniques like dropout, this is to prevent overfitting. Therefore, future research can be carried out on the wind direction to improve the accuracy of the model.

\section{REFERENCES}

[1] Ahrens CD, Henson R. Essentials of meteorology: An invitation to the atmosphere. Cengage Learning; 2016 Dec 5.

[2] DeFreitas NC, Silva MP, Sakamoto MS. Wind speeds forecasting: a review. Int. J. Eng. Res. Appl., 2018; 8:4-9.

[3] Larraondo PR, Inza I, Lozano JA. A system for airport weather forecasting based on circular regression trees. Environmental modelling \& software, 2018; Feb 1; 100:24-32.

[4] Rotich N. Forecasting of wind speeds and directions with artificial neural networks. 2014. Oct.

[5] Rozas-Larraondo P, Inza I, Lozano JA. A method for wind speed forecasting in airports based on nonparametric regression. Weather and Forecasting, 2014; 29(6):1332-42.

[6] Clark DA, Ferris RF, Moradi DD. Airport Wind Observations Architectural Analysis. Massachusetts INST of Tech Lexington; 2018 Jul 10 .

[7] Jung J, Broadwater RP. Current status and future advances for wind speed and power forecasting. Renewable and Sustainable Energy Reviews, 2014 Mar 1; 31:762-77.

[8] Früh WG. Evaluation of simple wind power forecasting methods applied to a long-term wind record from Scotland. In International Conference on Renewable Energies and Power Quality (ICREPQ'12), Santiago de Compostela 2012 Mar.

[9] Monfared M, Rastegar H, Kojabadi HM. A new strategy for wind speed forecasting using artificial intelligent methods. Renewable energy, 2009; Mar 1; 34(3):845-8.

[10] Demuth H, Beale M. Neural Network Toolbox User'S Guide: For Use with Matlab, Version 4

[11] Esen H, Inalli M, Sengur A, Esen M. Artificial neural networks and adaptive neuro-fuzzy assessments for ground-coupled heat pump system. Energy and Buildings, 2008; Jan 1; 40(6):1074-83.
[12] Shiruru, K. An Introduction to Artificial Neural Network. An international journal of Advance Research and Innovative Ideas in Education, 2016, 27.

[13] Du, K. L., \& Swamy, M. N. Neural networks and statistical learning. Canada: Springer Science \& Business Media, 2013 July.

[14] Wesonga R, Nabugoomu F, Ababneh F, Owino A. Simulation of time series wind speed at an international airport. Simulation, 2019; 95(2):171-84.

[15] Kaur T, Kumar S, Segal R. Application of artificial neural network for short term wind speed forecasting. In 2016 Biennial international conference on power and energy systems: towards sustainable energy (PESTSE) 2016 Jan 21 (pp. 1-5). IEEE.

[16] Fazelpour F, Tarashkar N, Rosen MA. Short-term wind speed forecasting using artificial neural networks for Tehran, Iran. International Journal of Energy and Environmental Engineering, 2016; 7(4):377-90.

[17] Hu Q, Zhang S, Yu M, Xie Z. Short-term wind speed or power forecasting with heteroscedastic support vector regression. IEEE Transactions on Sustainable Energy, 2015 Nov 23; 7(1):241-9.

[18] Hussin NH, Yusof F, Norrulashikin SM. Forecasting Wind Speed in Peninsular Malaysia: An Application of ARIMA and ARIMAGARCH Models. Pertanika Journal of Science \& Technology, 2021 Jan 1;29(1).

[19] Liu H, Tian HQ, Chen C, Li YF. An experimental investigation of two Wavelet-MLP hybrid frameworks for wind speed prediction using GA and PSO optimization. International Journal of Electrical Power \& Energy Systems, 2013; Nov 1; 52:161-73.

[20] Shi, J., Guo, J., \& Zheng, S. Evaluation of hybrid forecasting approach for wind speed and power generation time series. Journal of Renewable and Sustainable Energy Review, 2012;16:80-3471.

[21] Jung S, Kwon SD. Weighted error functions in artificial neural networks for improved wind energy potential estimation. Applied Energy, 2013 Nov 1; 111:778-90.

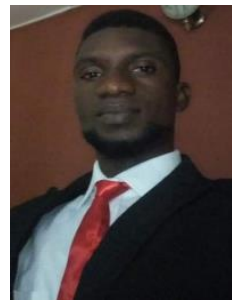

Lawrence Emmanuel is a graduate assistant in the Department of Computer Science Federal University Wukari, Taraba State Nigeria. He is an indigene of Ussa local government area of Taraba State, Nigeria. He Had a Bsc. in Computer Science from Federal University Wukari, Taraba State Nigeria in 2017. He is currently undergoing a master's degree in Computer Science in Modibbo Adama University, Yola Adamawa State, Nigeria. His research interest includes data science and

machine learning

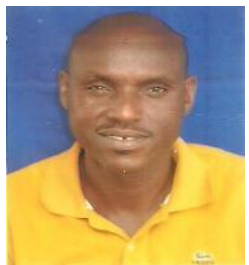

Yusuf Musa Malgwi is a Lecturer with the Department of Computer Science Modibbo Adama University, Yola, and Adamawa state-Nigeria. He graduated with a Bachelor of Technology in Computer Science with honours from the Federal University of Technology, Yola in 2006. M.Sc. Computer Science in 2014 from Adamawa State University, Mubi-Nigeria, and $\mathrm{PhD}$ in Computer Science in 2019 from Modibbo Adama University Yola Adamawa State- Nigeria.

He specializes in Machine Learning/Medical Informatics. The main courses of teaching and learning are in the area of Computer Science. He has supervised $5 \mathrm{PhD}$ candidates as Co-Supervisor and $9 \mathrm{M}$.Sc candidates to completion as the main supervisor. His experience in postgraduate supervision has been recognized as he was appointed the Postgraduate Cocoordinator for the Department of Computer Science of the Modibbo Adama University, Yola. He has also published more than 20 Journals in indexed publications especially those with high impact factors.

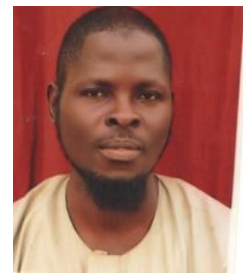

Moshood Abiola Hambali, is a lecturer in the Department of Computer Science, Faculty of Pure and Applied Sciences, Federal University Wukari, Wukari-

Taraba State, Nigeria. He graduated with a B. Sc. (Hons) in Computer Science from Al-Hikmah University, Ilorin; M. Sc. And PhD. in Computer Science Department from the University of Ilorin and Nigeria. He had published over 20 articles in both local and international journals. His research interest includes Data mining, Bio-Informatics, Machine Learning and Information Security. 